\title{
THE REALIZATION OF QUALIA, PERSONS, AND ARTIFACTS
}

BY

BEN WHITE

\begin{abstract}
This article argues that standard causal and functionalist definitions of realization fail to account for the realization of entities that cannot be individuated in causal or functional terms. By modifying such definitions to require that realizers also logically suffice for any historical properties of the entities they realize, one can provide for the realization of entities whose resistance to causal/functional individuation stems from their possession of individuative historical properties. But if qualia cannot be causally or functionally individuated, then qualia can be physically realized only if the thesis that all things are physical or physically realized is insufficient for physicalism.
\end{abstract}

\section{Introduction}

Philosophers often describe certain things as being 'realized' or 'realizable' by others. Mental states, e.g., are said to be realized by brain states; algorithms by the circuitry of the computers on which they are run; statues by the matter of which they are composed; and certain macro-physical properties, e.g. the hardness of a diamond, are sometimes said to be realized by the micro-physical states of the objects that bear them (Gillett, 2002, 2010). Despite (or perhaps because of) the wide variety of cases to which the notion of realization has been applied, there is as yet no common consensus as to how it is best defined. Two alternative conceptions of the relation emerge from the definitions that have been proposed thus far, one of which locates the nature of realization in one thing's deriving its distinctive causal profile from that of another, and the other of which views realization as instead consisting in the performance, by one thing, of another's distinctive

Pacific Philosophical Quarterly •• (2017) ••-•• DOI: 10.1111/papq.12217

(C) 2017 The Author

Pacific Philosophical Quarterly (C) 2017 University of Southern California and John Wiley \& Sons Ltd. 
function. I will argue that both these conceptions of realization fail to allow for the realization of entities, e.g. qualia, persons, and certain artifacts, that cannot be fully individuated in causal or functional terms. ${ }^{1}$

The article is structured as follows: Section 2 surveys some of the prominent definitions of realization currently on offer and takes the diversity among them to support a pluralistic treatment of realization, which views the term 'realization' as picking out a variety of distinct relations that share certain features in common. Section 3 argues that the definitions discussed in Section 2 fail to allow for the realization of qualia and certain entities (e.g. persons and certain artifacts) with individuative historical features. Section 4 then concludes the article by suggesting how one might allow for the realization of historically individuated entities in a manner consistent with physicalism, but argues that the postulation of the form of realization needed to provide for the realization of qualia renders the claim that all entities are physical or physically realized insufficient for physicalism.

\section{Causal and functionalist definitions of realization}

Realization is typically understood to be a binary, synchronic relation of dependence such that realized entities supervene on their realizers and realizers are moreover in some sense responsible for the occurrence or instantiation of the entities they realize. ${ }^{2}$ Attempts to give a more precise definition of the nature of the dependence that realization involves have tended to fall into one of two camps. The first of these construes realization as consisting in the derivation of one thing's distinctive causal profile from that of another. This causal or 'powers-based' conception of realization has itself been defended in two more specific forms. One of these is the 'causal-subset' conception of realization (hereafter 'C-S') advocated by Wilson (1999, 2011), Clapp (2001), and Shoemaker (2001, 2007). As formulated by Clapp (2001, p. 129), C-S holds that ' $P$ realizes $Q$ if and only if (def.), where $p$ and $q$ are the sets of powers constituting $P$ and $Q, q \subset p$.' On this proposal, the dependence of realized entities on their realizers is understood to consist in the fact that every causal power possessed by a realized entity is token-identical with some causal power of its realizer.

In addition to the kind of one-one relation described by C-S, one may also be interested in using the notion of realization to characterize the many-one relation that certain realized entities bear to certain properties of and relations between their various parts. ${ }^{3}$ In contrast to the kinds of cases that fall under the purview of C-S, in cases of the latter sort, the causal powers that the realized entity derives from its realizers are often different from those that its various realizers themselves possess. Such a case is discussed by Gillett (2010, p. 172), who notes that while the hardness of a diamond is 
plausibly viewed as realized by certain properties of and relations between its constituent atoms, the diamond's hardness is individuated by certain causal powers that none of its atomic-level realizers has or bestows. Whereas the former is individuated by the ability to scratch, without being scratched by, certain types of surfaces, the causal powers associated with the latter instead concern such things as the ability to form molecular bonds having a certain kind of structure, which remain intact under certain degrees and types of external stress. Although the possession of these causal powers by the diamond's constituent atoms is responsible for, and helps explain, the diamond's possession of the causal powers that are individuative of its hardness, the latter powers are nonetheless not identical with any of the former.

Reflection on such cases leads Gillett (2002, p. 322; 2003, p. 594) to propose an alternative powers-based definition of realization, which holds that '[p]roperty/relation instance(s) $F_{1}-F_{n}$ realize an instance of a property $G$, in an individual $s$, if and only if $s$ has powers that are individuative of an instance of $G$ in virtue of the powers contributed by $F_{1}-F_{n}$ to $s$ or $s$ 's constituent(s), but not vice versa.' Since Gillett's definition does not require the causal powers individuative of $G$ to be identical with those contributed by $F_{1}-F_{n}$, the relation he defines can hold in cases wherein the realized entity is individuated by causal powers that its realizers do not have. This distinguishes his definition of realization from that of C-S. All the same, Gillett's definition can still be seen to share with C-S the conception of realization as consisting in the derivation of one thing's distinctive causal profile from that of another.

The powers-based conception of realization endorsed by Gillett and proponents of C-S contrasts with an alternative conception that views realization as instead consisting in the performance by one thing of another's distinctive function. Proponents of the latter, functionalist conception of realization include Melnyk (2006) and Polger (2007, p. 251), who defines the relation as follows: 'Property/state instance $P$ realizes property/state instance $G$ iff $P$ has the function $\mathrm{F}_{G}(x)[$; i.e.] $P$ realizes $G$ if and only if $P$ has the $G$-function, if it plays the $G$-role.' Proponents of the functionalist conception of realization have objected to powers-based definitions of the relation on the grounds that not all of the sorts of things that are commonly spoken of as realizable have or bestow a distinctive set of causal powers. As Melnyk (2006, p. 146) notes, certain putatively realizable properties, e.g. 'having such-and-such a biological function' or (to use an example of Polger's (2007, p. 241)) being a genuine dollar bill, 'seem to be such that possession of them requires having not just causal powers but an actual causal history of some particular kind. ${ }^{4}$ Moreover, as Polger (2007, p. 241) points out, some of the most paradigmatic examples of realizable entities, e.g. Turing machines, do not appear to have or bestow any causal powers at all. Powers-based definitions like those of Gillett and Clapp 
cannot provide for the realization of such entities, because there are no unique sets of 'powers that are individuative [or constitutive] of' them.

This objection does not apply to the functionalist conception of realization advocated by Melnyk and Polger for the simple reason that not all functions are causal functions (Polger 2007, p. 251) ${ }^{5}$ Certain functions (e.g. biological, computational, or representational functions) are thus distinguished not (or not merely) by the causal powers they involve, but instead (or also) by the historical properties that the things that have them must exemplify, by the carrying out of certain formal operations on numbers or symbols, or by the possession of certain veridicality conditions. Consequently, if to be realized by $x$ is just to be individuated by a function that $x$ performs, then realized entities needn't be causally individuable. Unlike the proposals of Gillett and Clapp, the definitions of realization offered by Melnyk and Polger are hence able to treat entities like Turing machines and biological properties as realizable, so long as such things can be equated with or shown to have a distinctive function.

While this may seem to give the functionalist conception of realization a decisive advantage over its powers-based rival, advocates of the latter can reply that the definitions of realization they offer need not, and are indeed not intended to, capture the sorts of cases that Melnyk and Polger cite as counterexamples to their view. ${ }^{6}$ In support of this contention, one might follow Endicott (2012) and Gillett (2010, p. 167 fn5; 2013, pp. 166-9) in arguing that the notion of realization serves different explanatory roles, and hence has different meanings, in different theoretical contexts, and that there is, for this reason, no single realization relation, but rather a number of distinct relations that the term 'realization' can be legitimately used to refer to. The resulting pluralistic view of realization gives rise to the idea that the powers-based and functionalist conceptions of realization are not competing theories of a single relation, but rather attempts to identify two different kinds of relations that are explanatorily relevant in different contexts. If this is correct, though, then it is no objection to the powers-based definitions offered by Clapp and Gillett that they fail to treat entities like biological properties and Turing machines as realizable. For one cannot condemn a definition of one sense of realization for failing to accommodate cases that pertain to a different theoretical context.

Accepting this point forces us to recognize that even in its technical, philosophical usage, the term 'realization' is ambiguous, or at least polysemous, between realization as conceived under C-S (hereafter 'realization ${ }_{\mathrm{C}-\mathrm{S}}$ '), realization as conceived by Gillett (hereafter 'realization ${ }_{\mathrm{G}}$ '), and realization as conceived under the functionalist definitions endorsed by Melnyk and Polger (hereafter 'realization ${ }_{\mathrm{F}}$ ). ${ }^{7}$ Having admitted these various forms of realization as distinct but equally legitimate referents of the term, one would think that we should now be able to subsume any putative instance of 
realization under at least one of the relations distinguished thus far. The following section will argue, however, that there are certain entities, viz. qualia, persons, and certain artifacts, that seem physically realizable, but which are nevertheless not easily captured by any of the three forms of realization discussed above.

\section{The realization of qualia, persons, and artifacts}

Despite their differences, the definitions of realization discussed in the previous section share in common the view that realized entities must be capable of being individuated in purely causal and/or functional terms (hereafter ' $\mathrm{c} /$ f individuated'). ${ }^{8}$ The existence of realized entities that fail to meet this standard would hence entail the existence of a further form of realization that has yet to be defined. I suggest that qualia, persons, and certain artifacts are plausibly viewed as entities of this sort, for it seems reasonable to think that such entities are physically realized, yet there are also good grounds for thinking that they are not $\mathrm{c} / \mathrm{f}$ individuable.

In the case of qualia, there are a number of familiar arguments (e.g. the inverted qualia argument, Jackson's (1982) knowledge argument, Block's (1978) absent qualia argument, and Nagel's (1974) argument from the subjective character of experience) indicating that phenomenal experience involves certain intrinsic, qualitative features (qualia) that cannot be fully individuated in purely causal or functional terms. While such arguments are contentious, they nonetheless strike me as lending enough support to the hypothesis that qualia are not $\mathrm{c} / \mathrm{f}$ individuable to make it worth asking how qualia might be realized if that hypothesis is correct. My contention is that if that hypothesis is correct, then no theory of realization that treats realization $_{\mathrm{C}-\mathrm{S}}$, realization $\mathrm{G}_{\mathrm{G}}$, and realization $\mathrm{F}_{\mathrm{F}}$ as the only forms of realization that there are can provide for the realization of qualia. ${ }^{9}$

The grounds for denying that persons and artifacts are $\mathrm{c} / \mathrm{f}$ individuable derive from the supposition (supported by Kripkean considerations) that such entities have essential origins. If this is so, then persons and artifacts are at least partly individuated by certain historical properties that do not appear to be individuable in terms of the causal powers and functions that their bearers have or perform. ${ }^{10}$ Assuming, therefore, that persons and artifacts do have essential origins, and such entities are also physically realized, then it again seems that there must be an additional form of realization besides realization $_{\mathrm{C}-\mathrm{S}}$, realization $\mathrm{G}_{\mathrm{G}}$, and realization $\mathrm{F}_{\mathrm{F}}$ to account for this fact.

Such, then, are the problems that the apparent realizability of qualia, persons, and artifacts raise for any theory of realization that treats realization $_{\mathrm{C}-\mathrm{S}}$, realization $\mathrm{G}_{\mathrm{G}}$, and realization $\mathrm{F}_{\mathrm{F}}$ as the only forms of realization that exist. The remainder of this section considers some potential responses to these problems. 
The first response questions whether the essential origins of persons and artifacts really raise any difficulties for the claim that such entities are realized $_{F}$. As noted above, realization ${ }_{F}$ differs from realization ${ }_{C-S}$ and realization $_{\mathrm{G}}$ in that it allows for the realization of entities, e.g. the property of having a certain biological function or of being a genuine dollar bill, that are historically individuated. One might therefore wonder why the alleged fact that persons and artifacts are at least partly individuated by their origins should prevent them from being realized ${ }_{\mathrm{F}}$ as well.

Here it is important to understand what accounts for the realizability $y_{F}$ of those historically individuated entities that are realizable $\mathrm{F}_{\mathrm{F}}$. An entity that is realized $_{\mathrm{F}}$ is individuated by a function that its realizers $\mathrm{F}_{\mathrm{F}}$ have or perform. A realized $_{\mathrm{F}}$ entity is also historically individuated when its individuative function is such that only things with certain historical properties can have or perform it. Thus, the property of having the biological function of pumping blood is both historically individuated and realizable F $_{\mathrm{F}}$ because it is individuated by a function that can be possessed or performed only by something that is a descendant of a type of structure that was preserved under natural selection because it pumped blood. Likewise, the property of being a genuine dollar bill is both historically individuated and realizable $\mathrm{F}_{\mathrm{F}}$ because it is individuated by a certain economic function that can be possessed or performed only by things that have been printed and put into circulation by the US Treasury Department.

Persons and artifacts differ from those historically individuated entities that are realizable $\mathrm{F}_{\mathrm{F}}$ in that they are not individuable by any functions of this sort. If they were, then it should be possible to distinguish myself from a molecule-for-molecule duplicate of me, and the desk at which I'm sitting from a molecule-for-molecule duplicate of it, by appealing to some functions that this desk and I have that our respective duplicates lack. But what sort of functions could these be?

An answer to this question might be forthcoming in the case of artifacts (e.g. famous artworks) that have a special economic value attached to their origins, for such entities could perhaps be distinguished from potential duplicates by their possession of certain economic functions that only things with the requisite historical credentials can have or perform. Just as a genuine dollar bill is distinguished from a counterfeit replica by its having a certain economic function that only objects produced by the US Treasury Department can perform, the Mona Lisa, e.g., might thus be distinguished from a perfect forgery by the fact that it has a certain economic function (deriving from its value) that only the work actually produced by Da Vinci can perform. Such considerations may enable us to individuate certain specially valued artifacts by their economic functions, and thereby treat them as realizable $\mathrm{F}_{\mathrm{F}}$ in the same way that the property of being a genuine 
dollar bill is. This does nothing to help us, however, with those more lowly or mass-produced artifacts whose origins fail to endow them with any distinctive economic value. For such entities, as for persons, we still seem to be left without any way of capturing their individuative historical properties in purely functional terms. ${ }^{11}$

An alternative solution to this difficulty would be to simply broaden the notion of function so that having certain origins constitutes having a certain function. To have essential origins would then be equivalent to being individuated in terms of some such function, and the fact that an entity's origins are essential to it would consequently pose no obstacle to its realizability $_{\mathrm{F}}$. Such a conception of function is suggested by Melnyk's (2006, p. 129) definition of a 'functional type' as 'some type whose tokening just is the tokening of some or other type that meets a certain condition, C., 12 Since any tokening of the type of things that have certain origins 'just is' a tokening of the type of things that meet the condition of having those origins, having certain origins qualifies under this definition as having a certain function. The fact that persons and artifacts have essential origins may thus pose no obstacle to their realization ${ }_{\mathrm{F}}$ if the notion of function is construed as Melnyk suggests.

One might reasonably object, however, that if the notion of function is watered down so much that to have a function consists simply in 'meet[ing] a certain condition,' then the functionalist conception of realization ends up being too weak to be of much explanatory use. Note first that since each entity $x$ is the sole thing that meets the condition of being identical to $x$, every $x$ qualifies, under Melnyk's definition of function, as having a certain individuative function (viz. the function of being identical to $x$ ) that it alone performs. Since to realize $\mathrm{F}_{\mathrm{F}}$ something is to perform its distinctive function, Melnyk's definition of function thus yields the result that everything realizes $_{\mathrm{F}}$ itself simply by virtue of being the only thing that is identical to it. Yet surely if any form of realization is reflexive, this should not follow from so trivial a fact as that nothing is identical to a given thing besides itself.

Second, if, as Melnyk suggests, anything that meets a certain condition thereby qualifies as performing a function uniquely associated with the satisfaction of that condition, it follows that for every true proposition $p$, there is a distinctive function of being such that $p$ that everything performs and hence realizes $\mathrm{F}_{\mathrm{F}}$. For given any true proposition $p$, everything meets the condition of being such that $p$, and thus, according to Melnyk's definition of function, performs the function of being such that $p$. But if this is so, then each individual thing will realize $\mathrm{F}_{\mathrm{F}}$ an infinite number of things simply of by virtue of existing in a world at which an infinite number of propositions are true. At this point it becomes hard to resist the impression that instances realization ${ }_{F}$ have become too easy to come by, and that as a result, the notion of realization ${ }_{F}$ has lost its usefulness as a way of 
illuminating the relation between certain functionally individuated entities (e.g. Turing machines and biological properties) and the physical states or systems on which they depend. For our understanding of the relation, e.g., between the property of having the biological function of pumping blood and the matter that makes up a human heart is hardly improved by being told that it is the same sort of relation as that which holds between the latter and the 'function' of being such that $2+2=4$.

Some more substantive notion of function thus seems to be needed if the concept of realization $\mathrm{F}_{\mathrm{F}}$ is to be worth having. A reasonable proposal would be to restrict the kinds of functions relevant to realization ${ }_{F}$ to those that figure into some type of functional explanation in the sciences by requiring the function that individuates a realized $_{\mathrm{F}}$ entity to be of a sort that is recognized and made use of in some type of scientific explanation. ${ }^{13}$ Entities that are individuated by causal, biological, economic, or computational functions would thereby qualify as realizable $\mathrm{F}_{\mathrm{F}}$, inasmuch as their individuative functions are of types that are made use of in certain kinds of physical, chemical, biological, economic, or mathemati$\mathrm{cal} /$ computer-scientific explanations. The seemingly spurious functions countenanced by Melnyk (e.g. the 'function' of being identical with $x$, or of being such that $2+2=4$ ) would, on the other hand, play no role in determining what realizes $s_{F}$ what, since there is no type of scientific explanation that makes use of them. ${ }^{14}$

Adopting this restriction on the kinds of functions relevant to realization $\mathrm{F}_{\mathrm{F}}$ enables us to treat things like Turing machines, biological organs, and currency as realizable $\mathrm{F}_{\mathrm{F}}$ while also preventing the extension of realization $\mathrm{F}_{\mathrm{F}}$ from becoming so broad that the concept loses its explanatory use. Yet once the extension of realization ${ }_{\mathrm{F}}$ is pared down in this way, persons and certain artifacts will again fail to fall inside it. For aside from those specially valued artifacts that may perhaps be individuated by certain economic functions, persons and artifacts do not seem to be fully individuable in terms of any functions of a sort that are invoked in scientific explanations. This is because the origins of such entities are essential to them, but (specially valued artifacts aside) their having the origins they do does not seem to entail or be entailed by their possession or performance of any such function. As any other plausible restriction on the types of functions relevant to realization $_{F}$ seems likely to lead to the same result, the basic difficulty seems quite general: While we must impose certain constraints on the types of functions by which realized $\mathrm{F}_{\mathrm{F}}$ entities are individuated in order to keep realization $_{\mathrm{F}}$ from being drained of its explanatory use, persons and certain artifacts seem incapable of being individuated by their possession or performance of any functions that would satisfy these constraints. Consequently, if realization $_{F}$ is to be a concept worth having, then it appears that persons and certain artifacts must be deemed unrealizable $\mathrm{F}_{\mathrm{F}}$. 


\subsection{WILSON ON REALIZATION ${ }_{\mathrm{C}-\mathrm{S}}$}

An alternative way of allowing for the realization of persons, artifacts, and qualia without postulating any additional forms of realization besides realization $_{\mathrm{C}-\mathrm{S}}$, realization $\mathrm{G}_{\mathrm{G}}$, and realization $\mathrm{F}_{\mathrm{F}}$ might be sought in the view of realization ${ }_{\mathrm{C}-\mathrm{S}}$ endorsed by Wilson (2011). Wilson differs from other C-S theorists in holding that an entity need not be fully individuated by its causal powers in order to be realizable ${ }_{\mathrm{C}-\mathrm{S} .}{ }^{15}$ Thus, in contrast to Clapp (2001, p. 129), whose definition of realization ${ }_{\mathrm{C}-\mathrm{S}}$ requires both realized $_{\mathrm{C}-\mathrm{S}}$ entities and their realizers ${ }_{\mathrm{C}-\mathrm{S}}$ to be 'constitut[ed]' by 'sets of powers,' Wilson (2011, pp. 128-9) maintains that an entity's being realized $\mathrm{C}_{\mathrm{C}-\mathrm{S}}$ is 'compatible with [its] having a non-causal aspect not had by its [realizer ${ }_{\mathrm{C}-\mathrm{S}}$.' On her view, so long as $x$ 's causal powers constitute a proper subset of $y$ 's, $y$ realizes C-s $x$, even if $x$ also has certain essential features ('e.g., a non-causal quiddity or categorical aspect') that cannot be individuated in causal terms. While Wilson herself seems to hold that phenomenal features are causally individuable, ${ }^{16}$ the fact that her account of realization ${ }_{\mathrm{C}-\mathrm{S}}$ permits the realization $_{\mathrm{C}-\mathrm{S}}$ of entities with essential features that cannot be so individuated suggests that her account might allow us to treat qualia, persons, and artifacts as realizable $\mathrm{C}_{\mathrm{C}-\mathrm{S}}$ even if their essential phenomenal and historical features cannot be individuated in purely causal terms.

In treating entities that cannot be causally individuated as realizable $\mathrm{C}_{\mathrm{S}}$, however, Wilson's conception of realization ${ }_{\mathrm{C}-\mathrm{S}}$ fails to satisfy a condition that I think any form of realization ought to meet. ${ }^{17}$ This condition runs as follows:

In order for $y$ to realize $x$, the features of $y$ that make it a realizer of $x$ must make $y$ sufficient for the occurrence or instantiation of all of $x$ 's individuative features, and for any given individuative feature $F$ of $x$, the fact that $y$ suffices for $F$ must not be due solely to the fact that $y$ suffices for something other than $F$ that is itself sufficient for $F$.

In abbreviated form: each individuative feature of any realized entity must directly depend on its realizer. ${ }^{18}$ Since the assumption that $x$ 's causal powers constitute a proper subset of $y$ 's does not ensure that $y$ will also suffice for any non-causal individuative features that $x$ may have, Wilson's view of realization $_{\mathrm{C}-\mathrm{S}}$ allows us to treat something as realizable C-S $_{\text {S }}$ even if it is partly individuated by certain non-causal features that do not directly depend on its realizer $_{\mathrm{C}-\mathrm{S}}$. Her conception of realization $\mathrm{C}_{\mathrm{C}-\mathrm{S}}$ consequently fails to satisfy the condition just proposed and is therefore, I think, inadequate.

What reason do we have, though, to accept the above condition as a constraint on any adequate form of realization? Why should we require all the individuative features of any realized entity to depend directly upon its realizer? Why isn't it enough if only some of its features so depend? To begin, note that there is a clear distinction to be made between cases wherein $y$ 
realizes $x$ in its entirety, and cases wherein $y$ only realizes certain features or components of $x$. The properties of and relations between the atomic-level constituents of a gallon of water that realize ${ }_{\mathrm{G}}$ its transparency thus do not realize $_{\mathrm{G}}$ the water in its entirety, but only one of its features. Given this distinction, though, it would seem that whenever some of $x$ 's individuative features fail to directly depend on $y, y$ is best described not as realizing $x$ in its entirety, but rather as realizing only those features of $x$ that $d o$ depend directly on $y$. For if $y$ itself only suffices for some of $x$ 's individuative features, it is unclear what warrant we could have for claiming that $y$ realizes $x$ as a whole (including those individuative features of $x$ that $y$ does not directly suffice for), and not just those features of $x$ that $y$ does suffice to guarantee the occurrence or instantiation of. Thus, in any case where $x$ has certain noncausal individuative features, yet the causal powers that it does have constitute a proper subset of $y$ 's, $y$ seems best described not as realizing $\mathrm{C}-\mathrm{s} x$ in its entirety, but rather as realizing ${ }_{\mathrm{C}-\mathrm{S}}$ only certain features of $x$, viz. its causal powers. For while $x$ 's causal powers do directly depend on $y, x$ 's non-causal individuative features do not. If this is correct, then (pace Wilson) the assumption that qualia, persons, and artifacts cannot be causally individuated means that they themselves cannot be realized $\mathrm{C}_{\mathrm{S}}$; only their causal powers can.

The foregoing criticism of Wilson's account of realization ${ }_{\mathrm{C}-\mathrm{S}}$ bears some similarity to an objection raised by Melnyk (2006, pp. 138-43), who argues that theories of realization ${ }_{\mathrm{C}-\mathrm{S}}$ like Wilson's that allow for the realization $\mathrm{C}_{\mathrm{C}-\mathrm{S}}$ of entities that are not identical with clusters of causal powers are too weak to provide an adequate realization-based formulation of physicalism, because such theories fail to ensure (a) that all physically realized ${ }_{\mathrm{C}-\mathrm{s}}$ entities are fully constituted by their physical realizers, and (b) that all truths about such entities are made true by physical phenomena. ${ }^{19}$ With regard to (a), Melnyk contends that if $x$ has certain non-causal individuative features, then the assumption that $x$ 's causal powers constitute a proper subset of those of some physical entity $y$ does not ensure that $y$ fully constitutes $x$, for $y$ may fail to constitute $x$ 's non-causal features. With regard to (b), Melnyk contends that given $x$ 's partly non-causal nature, the assumption just mentioned may likewise fail to ensure that all truths about $x$ are made true by physical phenomena. For if $x$ is a property instantiated in some object $o$, then the ascription of $x$ to $o$ will be made true partly by the fact that $o$ has $x$ 's non-causal features. But the fact that $x$ 's causal powers constitute a proper subset of $y$ 's does not guarantee that the ascription of $x$ 's non-causal features to $o$ will itself be made true by physical phenomena.

Wilson (2011, p. 129; 2016, pp. 359-60) responds to Melnyk by arguing that her account's allowance for the realization ${ }_{\mathrm{C}-\mathrm{S}}$ of entities with non-causal individuative features does not undermine its suitability for use in formulating physicalism, because "truths about physical constitution or truthmaking, being broadly scientific truths, are neutral as regards whatever non-causal 
aspects of features there might be; hence the grounds of such truths must also be neutral on whether properties have non-causal aspects.' More generally, Wilson contends that 'any non-causal aspects of [a given realized $\mathrm{C}_{\mathrm{S}-\mathrm{S}}$ feature] $S$ are irrelevant to broadly scientific goings-on: scientific truths do not in any way depend on or otherwise track whether scientific features have non-causal aspects ... Hence that $S$ has such aspects (whether or not shared by $\left[S\right.$ 's realizer $\left.{ }_{\mathrm{C}-\mathrm{s}}\right] P$ ) cannot undermine $S$ 's physical acceptability, given $P$ 's physical acceptability.' Thus stated, Wilson's response can be seen to rest on the assumption that the only aspects of a feature that can bear on its compatibility with physicalism are those that are relevant to 'broadly scientific goings-on.' Given, then, that non-causal aspects are (allegedly) irrelevant to such goings-on, it follows that any 'non-causal aspects of $S$ are irrelevant to $S$ 's physical acceptability.'

While I find Wilson's response to Melnyk unconvincing, ${ }^{20}$ the question of whether her account of realization $\mathrm{C}_{-\mathrm{S}}$ is strong enough to yield an adequate formulation of physicalism is tangential to what I take to be its main problem. For although I agree with Melnyk that Wilson's allowance for the realization $\mathrm{C}_{\mathrm{S}}$ of entities with non-causal individuative features makes her account of realization $\mathrm{C}_{\mathrm{S}}$ too weak, the reason why it strikes me as too weak is not that it fails to yield an adequate formulation of physicalism (for even if it does fail in that respect, I see no reason to reject it on these grounds; notions of realization might be useful for other purposes besides just formulating physicalism). The problem, as I see it, is rather that since the fact that $x$ 's causal powers constitute a proper subset of $y$ 's does not guarantee that $y$ suffices for any of $x$ 's non-causal features, in cases where some of $x$ 's individuative features are non-causal in nature, it becomes unclear what warrant one could have for claiming that $y$ realizes $_{\mathrm{C}-\mathrm{S}} x$ in its entirety, and not just $x$ 's causal powers. For surely if realizers are to 'make real' what they realize, then in order for $y$ to realize $x, y$ must presumably suffice to guarantee the occurrence or instantiation of all those features that make $x$ what it is. Failing that, it appears that $y$ will at most 'make real' only part of $x$, which is to say that $y$ doesn't realize $x$ itself, but at most only some of its features. Despite Wilson's attempt to allow for the realization ${ }_{\mathrm{C}-\mathrm{S}}$ of entities that cannot be causally individuated, one is thus left with the impression that while the causal aspects of such entities can perhaps be realized $_{\mathrm{C}-\mathrm{S}}$, the entities themselves cannot.

\subsection{SHOEMAKER ON THE REALIZATION OF QUALIA}

Another way of accounting for the realization of qualia within the framework of a purely causal/functionalist conception of realization is suggested by Shoemaker (2007, p. 117), who maintains that 'while individual qualia are not functionally definable,' they nevertheless 'have causal profiles and can be physically realized.' Shoemaker works up to this conclusion by 
arguing that despite the functional indefinability of individual qualia, 'the relations of qualitative similarity and difference between experiences are functionally definable, and ... because of this the property of being a quale is functionally definable.' His argument for this claim rests on the premises (a) that to be a quale or phenomenal property ${ }^{21}$ is to be a feature of an experience by virtue of which that experience stands in relations of qualitative similarity and difference to other experiences, and (b) that relations of qualitative similarity and difference between experiences can be functionally defined in terms of the inductive tendencies and recognitional and discriminatory capacities of creatures that have them (Shoemaker, 2007, p. 121). This reasoning leads Shoemaker to propose that:

... a property [is] a quale if it belongs to a family of properties such that (1) experiences that are exactly alike with respect to which properties in this family they instantiate will be qualitatively identical, (2) experiences that differ in what properties they instantiate will be qualitatively different, and (3) all of the similarity and difference relations amongst experiences are determined by what properties in this family they instantiate (2007, p. 122).

With these conditions in place, Shoemaker (2007, p. 123) then claims that a particular quale $Q$ is realized by a physical property $P$ iff $P$ belongs to a family of physical properties $P_{1} \ldots P_{n}$ that satisfies conditions (1) and (3), and $P$ 's causal powers stand in the appropriate subset relations to those of $Q .^{22}$

Two questions strike me as central to the evaluation of Shoemaker's proposal. First, one might wonder why Shoemaker's functional definition of what it is to be a quale is needed to establish that qualia are realizable if the causal-subset relations that are in other cases sufficient for realization $_{\mathrm{C}-\mathrm{S}}$ can also hold between the respective causal powers of a quale and its putative physical realizer. Why isn't the supposed fact that a quale's causal powers stand in the appropriate subset relations to those of some physical property by itself enough to ensure that the former is realized $\mathrm{C}_{\mathrm{S}}$ by the latter? Second, one might ask whether qualia are supposed to be fully individuated by the causal powers that Shoemaker attributes to them. This question is made pertinent by the fact that, while granting that individual qualia cannot be functionally defined, Shoemaker (2007, pp. 119-20) also maintains that individual qualia have causal powers that are not contained in their functional description. This leaves open the possibility that for Shoemaker, individual qualia might be individuable in terms of their causal powers, despite the fact that they cannot be individuated in functional terms.

A satisfactory answer to the first of these two questions seems to presuppose a negative answer to the second. For if qualia are causally individuable, then it is unclear why Shoemaker's functional definition of what it is to be a quale should be needed to account for their realization. In that case, the fact that a physical property's causal powers bear the appropriate subset 
relations to those of some quale would seem enough by itself to make the former a realizer ${ }_{\mathrm{C}-\mathrm{S}}$ of the latter. By treating the causal-subset relations that are in other cases sufficient for realization ${ }_{\mathrm{C}-\mathrm{S}}$ as insufficient for the realization of qualia, Shoemaker thus seems to commit himself to the view that qualia are not individuable in terms of the causal powers they possess. ${ }^{23}$

This interpretation of Shoemaker's proposal enables us to answer the first question raised above by noting that if qualia are not fully individuated by their causal powers, then their realizers will have to satisfy further conditions beyond those that obtain in standard instances of realization ${ }_{\mathrm{C}-\mathrm{S}}$ in order to ensure that a quale's non-causal individuative features directly depend on its realizer. Shoemaker's requirement that the physical realizer of a quale belong to a family of properties on which relations of qualitative similarity among experiences depend may be viewed as a condition of this very sort. For assuming, as seems plausible, that the non-causal individuative features of qualia are distinguished by the non-causal relations of qualitative similarity among experiences that are invoked in Shoemaker's functional definition of what it is to be a quale, ${ }^{24}$ to require that the physical realizer of a quale belong to a family of properties on which such relations depend is to make the non-causal individuative features of any quale dependent upon its physical realizer.

Our evaluation of Shoemaker's account begins with the observation that since the causal-subset relations that are typically sufficient for realization $_{\mathrm{C}-\mathrm{S}}$ are, on his view, insufficient for the realization of qualia, the relation he defines as holding between qualia and their realizers is distinct from realization ${ }_{\mathrm{C}-\mathrm{S}}$. For whereas realizers ${ }_{\mathrm{C}-\mathrm{S}}$ need only stand in the appropriate causal-subset relations to the entities they realize $\mathrm{C}_{\mathrm{C}-\mathrm{S}}$, the physical realizers of qualia must, on his account, also belong to a family of properties on which relations of qualitative similarity among experiences depend. Since this further condition is also inessential to both realization $\mathrm{G}_{\mathrm{G}}$ and realization ${ }_{F}$, the relation that Shoemaker treats qualia as bearing to their realizers appears in fact to be a fourth form of realization distinct from the three discussed thus far. In this way, Shoemaker's proposal seems actually to imply one of the central theses of this article: viz. that no theory of realization that treats realization $\mathrm{C}-\mathrm{S}_{\mathrm{S}}$, realization ${ }_{\mathrm{G}}$, and realization $\mathrm{F}$ as the only forms of realization there are can account for the realization of qualia.

Despite the support it may thus lend to one of this article's central claims, Shoemaker's proposal still fails, I think, to provide a satisfactory account of how qualia are realized. This is because his requirement that the physical realizer $P$ of a quale $Q$ belong to a family of properties on which relations of qualitative similarity among experiences depend does not ensure that $Q$ 's non-causal individuative features directly depend on $P$. Consequently, on Shoemaker's proposal, the only features of $Q$ that are guaranteed to directly depend on $P$ are those of its causal features that $P$ suffices for by 
virtue of standing in the causal-subset relations that Shoemaker claims must obtain between $P$ and $Q$. As argued in the previous sub-section, though, all this entitles us to say is that $P$ realizes $Q$ 's causal features, not $Q$ itself, in its entirety.

In response to this criticism, one might argue that if, as suggested above, the non-causal individuative features of qualia can be distinguished by relations of qualitative similarity among experiences, and such relations are in turn determined by the physical realizers of qualia, then it would seem that the non-causal individuative features of qualia may directly depend upon their physical realizers after all. The problem with this line of reasoning is that the first conjecture is false: first appearances notwithstanding, the non-causal individuative features of qualia cannot be fully distinguished by relations of qualitative similarity among experiences. ${ }^{25}$ To see why, consider the possibility of an 'alien' color space $\mathbf{C}^{*}$ (i.e. a color space composed entirely of color qualia that we are unacquainted with) that is completely isomorphic to our own color space $\mathbf{C}$ in the sense that (a) each color quale $Q_{i}$ in $\mathbf{C}$ corresponds to a single 'alien' color quale $Q_{i}{ }^{*}$ in $\mathbf{C}^{*}$ such that, for any other quale $Q_{n}$ in $\mathbf{C}$ and its corresponding quale $Q_{n}{ }^{*}$ in C*, $Q_{i}$ is just as qualitatively similar to $Q_{n}$ as $Q_{i}{ }^{*}$ is to $Q_{n}{ }^{*}$, and (b) each quale $Q_{i}$ in $\mathbf{C}$ is just as qualitatively similar to each of the various qualia $Q_{1}{ }^{*} \ldots Q_{n}{ }^{*}$ in $\mathbf{C}^{*}$ as its corresponding quale $Q_{i}{ }^{*}$ is to the various qualia $Q_{1} \ldots Q_{n}$ in $\mathbf{C}$ to which $Q_{1}{ }^{*} \ldots Q_{n}{ }^{*}$ each correspond. Since the qualia making up such an alien color space would bear the same relations of qualitative similarity to one another and to the qualia that make up our own color space as the color qualia we are acquainted with bear to one another and to the qualia that constitute $\mathbf{C}^{*}$, it would be impossible for anyone to tell which of the two color spaces is our own solely on the basis of the relations of qualitative similarity among the qualia of which each space is composed. Considerations of qualitative similarity would be equally insufficient to enable one to tell whether any individual color quale were an alien quale or a member of our own color space, as every color quale $Q$ that we are acquainted with would have an alien 'twin' in $\mathbf{C}^{*}$ that bears the same relations of qualitative similarity to all the other qualia in $\mathbf{C}^{*}$ that $Q$ bears to all the other qualia in $\mathbf{C}$, and the same relations of qualitative similarity to the qualia in $\mathbf{C}$ that $Q$ bears to those in $\mathbf{C}^{*}$.

The possibility of an alien quality space of this sort shows that the non-causal individuative features of qualia cannot be fully distinguished by relations of qualitative similarity among experiences. For given two distinct but isomorphic quality spaces such as $\mathbf{C}$ and $\mathbf{C}^{*}$, one could have complete knowledge of all the relations of qualitative similarity that a certain member $Q$ of $\mathbf{C}$ and its corresponding 'twin' $Q^{*}$ in $\mathbf{C}^{*}$ enter into without being able to tell whether a certain quale $Q_{\text {? }}$ that one is acquainted with is identical to $Q$ or $Q^{*}$, even if one knows that $Q_{\text {? }}$ is in fact identical with one 
of these two qualia. To answer this question, one seems to require some additional knowledge of 'what it is like' to have experiences in which $Q$ or $Q^{*}$ are instantiated. Only then will one be able to say for certain which of these two qualia $Q_{\text {? }}$ is identical with, by determining whether 'what it is like' to experience $Q_{\text {? }}$ is the same as or different from 'what it is like' to experience $Q$ (or $Q^{*}$ ). Such considerations suggest that if qualia indeed have certain non-causal individuative features, then such features can be fully distinguished only on the basis of subjective experience. Functionally definable relations of qualitative similarity are inadequate to the task. ${ }^{26}$

If the foregoing reasoning is sound, then the supposition that the physical realizer $P$ of a quale $Q$ belongs to a family of physical properties that satisfies conditions (1) and (3) above fails to ensure that $P$ suffices for the non-causal individuative features that distinguish $Q$ from any potential alien 'twin' $Q^{*}$. The same is true of the supposition that $P$ and $Q$ 's respective causal powers are related in the way Shoemaker requires, for the fact that $P$ 's causal powers bear certain subset relations to $Q$ 's does not render $P$ sufficient for $Q$ 's non-causal features. As these are the only two constraints that Shoemaker places on the physical realizers of qualia, his account does not ensure that such realizers suffice for all the individuative features of the qualia they purportedly realize, and thus fails to satisfy the condition on realization proposed in Section 3.2.

\section{Realization and physicalism}

The results of the previous section suggest that if, as seems plausible, qualia, persons, and certain artifacts cannot be $\mathrm{c} / \mathrm{f}$ individuated, then none of the forms of realization discussed thus far provides an adequate account of the realization of such entities. Our discussion of the views of Melnyk, Wilson, and Shoemaker has indicated, moreover, that this conclusion cannot be avoided (a) by broadening the notion of function so that there is a distinctive function corresponding to every condition that a thing might meet, (b) by making the realization of a thing's causal features sufficient for the realization of that thing in its entirety, or (c) by suggesting that by determining relations of qualitative similarity among experiences, the physical realizers of qualia suffice for the non-causal individuative features of the qualia they realize. It appears, therefore, that if qualia, persons, and artifacts are indeed realizable, then some additional form of realization is needed to account for this fact.

Call a form of realization realization ${ }_{R}$ 'physicalist' or 'sufficient for physicalism' iff the thesis that all things are physical or physically realized $_{R}$ entails that physicalism is true, and 'non-physicalist' or 'insufficient for physicalism' iff this is not the case. This last section will argue 
that while a physicalist form of realization can be defined that may account for the realization of persons, artifacts, and other historically individuated entities, if qualia indeed cannot be $\mathrm{c} / \mathrm{f}$ individuated, then only a non-physicalist form of realization seems capable of accounting for their realization.

Starting, then, with persons and artifacts, the main reason that the forms of realization discussed thus far fail to provide a satisfactory account of the realization of such entities is that the conditions that they require realizers to meet do not guarantee that the realizers of persons and artifacts will suffice for the latter's individuative historical features. A quick solution to this problem can, however, be obtained by simply adding on to the conditions associated with each of these forms of realization the further requirement that a realizer's historical properties be logically sufficient for any historical properties that the entity it realizes has. Let realization $\mathrm{R}_{\mathrm{R} / \mathrm{H}}$ denote the modified form of realization that results from the inclusion of this additional, historical condition in the definition of any form of realization realization ${ }_{R}$. An entity $y$ may then, e.g., be said to realize $\mathrm{C}_{\mathrm{C}-\mathrm{S} / \mathrm{H}}$ another entity $x$ iff $y$ and $x$ 's respective causal powers stand in the subset relations required in order for $y$ to realize $\mathrm{C}_{\mathrm{S}-\mathrm{S}} x$ and $y$ 's historical properties are also logically sufficient for any historical properties that $x$ has; similarly, mutatis mutandis, for the other forms of realization canvassed above. With this modification in place, the historical properties of any realized entity are guaranteed to directly depend upon the historical properties of its realizer, thereby enabling the powers-based and functional definitions of realization distinguished in Section 2 to accommodate the realization of entities with individuative historical properties. So long, moreover, as those historical properties of a realizer that enable it to logically suffice for the historical properties of the entity it realizes do not entail the existence of any irreducible, non-physical entities, the incorporation of the historical condition on realization proposed above will not render any form of realization insufficient for physicalism that was not so before. Insofar, then, as realization ${ }_{\mathrm{C}-\mathrm{S}}$, realization ${ }_{\mathrm{G}}$, and

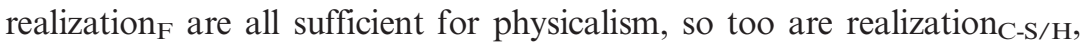
realization $_{\mathrm{G} / \mathrm{H}}$, and realization $\mathrm{F}_{\mathrm{H}}$ (provided that the condition mentioned in the previous sentence is satisfied).

The situation is different, however, with respect to the realization of qualia. For since the individuative features of qualia that prevent them from being $\mathrm{c} / \mathrm{f}$ individuated are not historical in nature, the historical condition proposed above will be of no use in defining a form of realization that allows for the physical realization of qualia. Moreover, since (in contrast to the individuative historical features of persons and artifacts) the non-causal/ non-functional individuative features of qualia do not seem to follow logically from any properties that their presumptive physical realizers might possess, ${ }^{27}$ the only apparent way that the physical realizers of qualia could 
be guaranteed to suffice for those features would be for there to be some (non-logical) law under which the occurrence of a quale's realizer necessitates the non-causal/non-functional individuative features of the quale it realizes. If this is correct, then the only way for a definition of realization to account for the physical realization of qualia in a way that ensures that a quale's individuative features all depend on its realizer will be for it to include a condition requiring realizers to be nomologically sufficient for any individuative features of the entities they realize that they do not logically suffice for. Only thus will those individuative features of qualia that cannot be fully defined in causal or functional terms be guaranteed to depend on their physical realizers. ${ }^{28}$

These considerations suggest that in order for qualia to be physically realized, there must be some form of realization realization ${ }_{\mathrm{R}}$ such that $y$ may realize $_{\mathrm{R}} x$ even if $y$ is only nomologically sufficient for certain of $x$ 's individuative features. Such a form of realization cannot, however, be sufficient for physicalism. For as many writers have pointed out, the claim that the physical facts are at least nomologically sufficient for all the facts is compatible with the existence of physicalistically unacceptable, emergent entities, which physical events suffice for only by virtue of certain irreducible, non-physical laws (Wilson, 1999; Melnyk, 2006, p. 133). Given, then, that the only kind of realization relation that seems capable of accounting for the realization of qualia is one that allows realizers to be merely nomologically sufficient for some of the individuative features of the things they realize, it follows that no form of realization will be able to account for the realization of qualia unless it allows for physicalistically unacceptable, emergent entities to be realized as well. Consequently, if qualia are physically realized, then the claim that all things are either physical or physically realized is insufficient for physicalism.

In conclusion, our examination of the powers-based and functional definitions distinguished in Section 2 suggests that realization ${ }_{\mathrm{C}-\mathrm{S}}$, realization ${ }_{\mathrm{G}}$, and realization ${ }_{\mathrm{F}}$ are all incapable of accounting for the realization of entities that cannot be $\mathrm{c} / \mathrm{f}$ individuated. As this section has shown, though, one can provide for the realization of entities, e.g. persons and certain artifacts, whose inability to be $\mathrm{c} / \mathrm{f}$ individuated is attributable to their possession of certain individuative historical features by modifying the definitions of realization $_{\mathrm{C}-\mathrm{S}}$, realization $\mathrm{G}_{\mathrm{G}}$, and realization $\mathrm{F}_{\mathrm{F}}$ to require the historical properties of realizers to logically suffice for any historical properties of the entities they realize. Yet if qualia cannot be $\mathrm{c} / \mathrm{f}$ individuated, then it seems that the only way to allow for the physical realization of qualia is to permit realizers to be merely nomologically sufficient for certain individuative features of the entities they realize. Since, however, no notion of realization that allows realized entities to depend with mere nomological necessity on their realizers can suffice for physicalism, it appears that if qualia cannot be $\mathrm{c} / \mathrm{f}$ 


\title{
individuated, then qualia can be physically realized only if the thesis that all things are physical or physically realized is insufficient for physicalism. ${ }^{29}$
}

\author{
Department of Philosophy, Temple University
}

\section{NOTES}

1 An anonymous referee worries that the novelty of this thesis may be undermined by the alleged fact that since "[a]11 realization theorists hold that being a realizer requires a thing or its parts to stand in some...causal relations or some broader range of "functional" relations [to the thing(s) it realizes,]...they all agree that if there were things that are not individuated as such, they would not be realizable.' The latter claim is, however, false. For as shown in Section 3 below, some realization theorists (e.g. Wilson and possibly Shoemaker) maintain that an entity can be realized even if it has certain essential features that cannot be fully individuated in purely causal or functional terms. One of the primary aims of this article is to show that standard causal and functionalist definitions of realization do not provide an adequate basis for the defense of such claims.

2 Some might want to refrain from claiming that realization is also asymmetric and irreflexive in order to allow for the possibility that certain fundamental entities realize themselves (Shoemaker, 2001, p. 85; 2007, p. 23). Note also that if causation is diachronic, then the dependence of realized entities on their realizers must be non-causal.

3 Thanks to an anonymous referee for stressing the advantages of framing the difference between C-S and Gillett's definition of realization in these terms.

4 Shoemaker $(2007$, p. $50 \mathrm{fn} 11)$ attempts to resolve this difficulty by suggesting that historically individuated entities may be realized by 'temporally extended states of affairs,' or by properties that things have 'in virtue of [their] career[s] embedding a [temporally extended] state of affairs of [a certain] type.' Neither of these proposals seems to me to address the problem raised above. For if some realized entities are at least partly individuated by their histories, and an entity's history is not determined by its causal powers (as indicated by the fact that entities with different histories can nevertheless have the same causal powers), then it follows that theories of realization that require realized entities to be fully individuated or constituted by sets of powers that they share with or derive from their realizers will fail to capture some instances of realization. Extending or otherwise augmenting the realizer of a given historically individuated entity $e$ to include certain historical properties, facts, or events that are relevant to $e$ 's individuation does nothing to change this fact. (Wilson's [2011] version of C-S circumvents this objection by omitting the requirement that realized entities be fully individuated or constituted by the causal powers they share with their realizers. Her position is criticized on other grounds, however, in Section 3.2 below.)

5 An anonymous referee suggests that causal theorists might respond by claiming that the term 'function' is ambiguous between abstract, mathematical functions, which are unsuited to be realized and thus needn't be captured by their account, and functions that specify a role that must be implemented, which their account does capture insofar as all such roles can be individuated in purely causal terms. While I am doubtful that all realized functional roles can indeed be causally individuated, I nevertheless take this suggestion to be consistent with the view defended below; viz. that the term 'realization' is itself ambiguous or polysemous between different conceptions of realization that are applicable to different types of cases.

6 Note also that there are some apparent instances of realization that Gillett's definition does capture but that Melnyk and Polger's do not; viz. instances wherein a realized entity is individuated by causal powers that its realizers do not have.

7 The term 'realization' has also been used by Lewis (1972) to describe the relation between a Ramsey sentence and the entities that satisfy it. Endicott (2012, pp. 42-3) locates this usage 
within a more general 'representational' tradition of realization, which treats realization as 'a semantic or intentional relation' between a representation and something that satisfies it. I will largely disregard this sense of realization in this article, as it strikes me as more or less equivalent with the already well-established notion of one thing's 'satisfying' or 'being a model (in the mathematical sense) of' another.

8 Not everyone would agree with this. Wilson (2011), e.g., denies that realized ${ }_{\mathrm{C}-\mathrm{S}}$ entities must be $\mathrm{c} / \mathrm{f}$ individuable. Her version of $\mathrm{C}-\mathrm{S}$ will be discussed below.

9 I stress that the thesis that qualia cannot be $\mathrm{c} / \mathrm{f}$ individuated will be taken as established by the arguments of Jackson (1982), Block (1978), Nagel (1974), et al. in what follows. Responding to the numerous counterarguments to this thesis is a task beyond the scope of the present article, whose principal aim is merely to show that if qualia, persons, and artifacts cannot $\mathrm{be} \mathrm{c} / \mathrm{f}$ individuated, then they cannot be realized in any of the three ways distinguished above. That said, in response to the suggestion (made by Shoemaker [1975], Wilson [2016, p. 360 fn15], and an anonymous referee) that qualia may be more susceptible to $\mathrm{c} / \mathrm{f}$ individuation if one allows the causal/functional profile of a given quale to include such 'qualitatively loaded' (the referee's term) features as the ability to cause awareness of certain qualitative aspects of our experience, or the belief that one's current experience has a certain qualitative character, one might object that such an approach risks leaving us without any means of determining which individuative descriptions correspond to which qualia. For if, e.g., a given color quale $Q_{1}$ is to be distinguished from all other entities in part by the fact that it causes awareness of $Q_{1}$, the same will presumably be equally true (mutatis mutandis) of most any other color quale as well. Yet if the powers that are cited in the individuative descriptions of a given set of qualia as distinguishing the members of that set from one another all involve ineliminable reference to the very qualia that they are supposed to help individuate, and all the qualia in that set share these same kinds of self-referential powers, then there seems to be no way of determining which individuative descriptions correspond to which qualia. Thus, in the case of color qualia, if the only features of $Q_{1}$ that distinguish it from some other color quale $Q_{2}$ are the same sort of self-referential powers that distinguish $Q_{2}$ from $Q_{1}$ (so that whereas $Q_{1}$ has the power to cause awareness of $Q_{1}, Q_{2}$ has the power to cause awareness of $Q_{2}$, and likewise for any other powers that are cited as distinguishing $Q_{2}$ from $Q_{1}$ ), then even if we are told that one of these two qualia is phenomenal red and the other is phenomenal green, their individuative descriptions would not enable us to determine which is which. Here it is important to bear in mind that in offering an individuative description of phenomenal red that distinguishes it from phenomenal green, one cannot simply advert to the fact that phenomenal red is unlike phenomenal green in that it causes awareness of phenomenal red as opposed to phenomenal green, for such a statement presupposes the very sort of distinction between phenomenal red and phenomenal green that an individuative description of phenomenal red is supposed to make clear. (Shoemaker [1975, p. 310] grapples with a similar problem, but ultimately concedes that 'at least some qualitative states (and qualitative beliefs) cannot be functionally defined.' His views are discussed at greater length in Section 3.3. below.)

${ }_{10}$ I stress that the thesis that persons and artifacts have essential origins and are for this reason resistant to $\mathrm{c} / \mathrm{f}$ individuation will be largely assumed in what follows. In response, however, to an anonymous referee's suggestion that having certain origins may carry certain causal consequences, e.g. making you 'such that your mother loves you more than [a molecule-for-molecule] duplicate' of you, it may first be noted that it seems odd to view your mother's love for you as an effect that you causally produce in her by virtue of your origins. If anything, this would instead seem to be caused by your mother's belief that you are her child. Your origins would thus seem insufficient to cause your mother to love you more than your duplicate if she somehow came to believe that she had no children. Perhaps this just shows that certain of the causal powers that one's origins endow one with are such that they are only manifested in conditions wherein one's origins are known to certain agents (so that, e.g., your origins would enable you to produce certain emotional responses or other effects in your mother that your duplicate could 
not in situations wherein your mother is in a position to know that she gave birth to you and not your duplicate). Even if an entity's origins do endow it with such powers, though, it is unclear that these powers would suffice to distinguish any potential duplicates whose origins are extremely similar (e.g. two perceptually indistinguishable red rubber balls produced seconds apart in a factory that makes thousands of such balls a day). And aside from a prior commitment to the view that everything is $\mathrm{c} / \mathrm{f}$ individuable, what reason do we have to think that the slightest difference in the origins of two molecule-for-molecule duplicates must always be reflected in some difference in their respective functions or causal powers? One who is committed to such a view might of course maintain that the absence of any difference in the causal or functional features of two distinct entities just shows that any differences in their respective origins are irrelevant to their individuation. Yet I see no obvious contradiction or impossibility in the thought of two entities sharing all the same causal and functional features while nevertheless having different essential origins.

11 An anonymous referee points out that there are other forms of value besides economic value, e.g. 'the value associated with token individuality or experiences.' I am doubtful, however, that such non-economic forms of value can be equated with the possession of a distinctive function in the way that having a certain economic value can, for it is unclear to me what sort of function we could attribute to an entity that would constitute its having such value. This distinguishes the Mona Lisa example from the mother-child example discussed in note 10, as the origin-dependent value that a child has to his/her mother does not seem to me to be functionally definable in the way that the origin-dependent economic value of the Mona Lisa is. (Thanks to an anonymous referee for pressing me to clarify the difference between these two cases.) If all children can be shown to have a certain distinctive function that derives from the value that they have to their mothers, and it can moreover be shown that the origins of any person are identifiable in terms of their value to their mother, then it may be possible to treat persons as realizable ${ }_{\mathrm{F}}$ along the lines suggested for the Mona Lisa above. However, both conjuncts in the antecedent of this conditional strike me as dubious.

12 Melnyk's notion of function is closely related to the view (criticized extensively by Gillett [2013]) that functions are second-order properties, specified by Ramsey sentences, which are 'realized' by whatever satisfies the description that their individuative Ramsey sentence contains. Such a view makes realization ${ }_{F}$ equivalent to the 'representational' form realization mentioned in note 7 .

13 Shoemaker (2007, pp. 64-71) suggests rather that 'the functional/non-functional distinction is best seen as a distinction between different sorts of concepts, not one between different sorts of properties.' On his view, to be a functional property is just to be a property picked out by a functional concept, i.e., a concept that 'do[es] not rule out the possibility that the propert[y it] pick[s] out ha[s] realizers that are causally diverse.' I see no reason, however, why those properties whose realizers are (or could be) causally diverse shouldn't be classified as functional regardless of how they are conceptualized. Many properties that are cited in functional explanations in the sciences would likely qualify as functional in this sense.

14 An anonymous referee suggests that even if functions like being such that $2+2=4$ play no role in the explanation of contingent facts, they might still play some role in the explanation of certain non-contingent facts, e.g. the fact that ' $2+2=4$ ' is necessarily true. The thought here seems to be that the necessity of a given proposition $p$ might be explained by the fact that nothing can exist without realizing the function of being such that $p$. This, however, just strikes me as another way of putting the already familiar point that necessary propositions are those that are true in all possible worlds. I therefore don't see what the reference to the function of being such that $2+2=4$ or the realization of that alleged function adds to our understanding of why ' $2+2=4$ ' is necessary.

15 This difference is largely due to the fact that other C-S theorists (e.g. Clapp [2001] and Shoemaker [2007, pp. 142-4]) endorse a causal theory of properties, according to which properties are fully individuated by the causal powers they bestow on their bearers. 
16 Wilson's stance on this issue seems to derive from her conviction that causal powers can include such 'qualitatively loaded' features as, e.g., the ability 'to produce awareness of qualitative aspects in experiencing subjects' (2016, p. $360 \mathrm{fn} 15)$. Thanks to an anonymous referee for pointing this out.

17 Strictly speaking, this condition applies only to what Shoemaker (2007, pp. 21-2) calls 'total' realization (in contrast to 'core' realization).

18 The requirement that the dependence of realized entities on their realizers be direct is meant to rule out seemingly spurious cases of realization wherein the putative 'realizer' of an entity $x$ is only indirectly sufficient for certain individuative features of $x$, in the sense that it suffices for those features only by virtue of realizing some other entity that is itself nomologically sufficient for them.

19 Melnyk notes that such theories will also fail to ensure that all physically realized ${ }_{\mathrm{C}-\mathrm{S}}$ entities are necessitated 'in the strongest' sense by their physical realizers (a condition that he likewise takes as necessary for physicalism), unless one stipulates that any non-causal features possessed by such entities are themselves necessitated 'in the strongest sense' by their causal powers.

20 Are scientific truths really as indifferent to non-causal aspects as Wilson claims? Wilson frequently refers to 'non-causal quiddities' as a potential example of such aspects, which suggests that non-causal haecceities might be included among such aspects as well. Such haecceities might be thought to distinguish one token fundamental particle from another particle of the same type. Quantum mechanics suggests, however, that there is in principle no way of distinguishing between two particles of the same type once their wave functions overlap. Here, then, we seem to have a case wherein certain scientific truths (viz. those dealing with quantum indistinguishability) depend very much on 'whether scientific features have non-causal aspects' (viz. on whether different particles of the same type can be distinguished by non-causal haecceities). (Teller [1998, p. 135] thus argues that 'there is a conceptually important difference in describing the facts of quantum mechanics with and without haecceities.') Moreover, even if non-causal aspects are irrelevant to 'broadly scientific goings-on,' why should this entitle physicalists to treat such aspects as irrelevant to the truth or falsity of physicalism (especially if (pace Wilson, 2016, p. $360 \mathrm{fn} 15$ ) such aspects include things like the phenomenal features of conscious states)?

21 I will use these terms interchangeably.

22 The requirement that $P_{1} \ldots P_{n}$ also satisfy (2) is omitted in order to allow for the possibility that $Q$ is multiply realizable. The causal-subset relations that Shoemaker requires to hold between $P$ and $Q$ are also a bit more complex than those involved in other accounts of realization ${ }_{\mathrm{C}-\mathrm{S}}$, but these added complications will have no major bearing on our discussion.

23 On the causal theory of properties that Shoemaker (2007, pp. 142-4) favors, though, it would seem that qualia must be causally individuable. The question of the causal individuability of qualia may thus constitute a point of tension in Shoemaker's position.

24 It is unclear whether Shoemaker would assent to this, given his denial that individual qualia are functionally definable. See note 25 .

25 This conclusion may indeed already follow from Shoemaker's (2007, p. 117) concession that 'individual qualia are not functionally definable.' For if relations of qualitative similarity among experiences are functionally definable, as Shoemaker claims, and the non-causal individuative features of individual qualia can in turn be distinguished by such relations, then there would seem to be nothing to prevent individual qualia from being functionally definable as well (unless one holds that qualia also have certain individuative causal features that are not contained in their functional description).

26 Note that the alien qualia that the foregoing argument appeals to differ from both 'absent' qualia and the qualia in an inverted quality space in that they are conceived as real qualitative features of the experiences of certain potential subjects, but are not identical to any of the qualia experienced by normal humans. The foregoing argument therefore bypasses controversies surrounding the possibility of 'absent' qualia (Block, 1980, and Shoemaker, 1975, 1981) and objections to the possibility of a functionally undetectable spectral inversion that advert to 
certain asymmetries in our phenomenal color space (Hardin, 1988, pp. 134 42). Thanks to an anonymous referee for drawing my attention to the need to make this clearer.

27 Aside, that is, from the property, if it can be deemed such, of being the realizer of a quale with such-and-such features.

28 An anonymous referee points out that qualia could still be metaphysically necessitated by their realizers even if their realizers do not logically suffice for them (if, e.g., the laws that render a quale's realizer sufficient for the quale it realizes are themselves metaphysically necessary). As I argue elsewhere, however, the claim that qualia and all other 'higher-level' properties are metaphysically necessitated by their physical bases in this way is compatible with certain forms of dualism, and thus insufficient for physicalism (White, 2017). The conclusions drawn in the following paragraph are therefore unaffected by the referee's observation.

29 Many thanks to two anonymous referees for their helpful comments.

\section{REFERENCES}

Block, N. (1978). 'Troubles with Functionalism,' Minnesota Studies in the Philosophy of Science 9, pp. 261-325.

Block, N. (1980). 'Are Absent Qualia Impossible?' Philosophical Review 89, pp. 257-274.

Clapp, L. (2001). 'Disjunctive Properties: Multiple Realizations,' Journal of Philosophy 98, pp. 111-136.

Endicott, R. (2012). 'Resolving Arguments by Different Conceptual Traditions of Realization,' Philosophical Studies 159, pp. 41-59.

Gillett, C. (2002). 'The Dimensions of Realization: A Critique of the Standard View,' Analysis 62 , pp. 316-323.

Gillett, C. (2003). 'The Metaphysics of Realization, Multiple Realizability, and the Special Sciences,' The Journal of Philosophy 100, pp. 591-603.

Gillett, C. (2010). 'Moving beyond the Subset Model of Realization: The Problem of Qualitative Distinctness in the Metaphysics of Science,' Synthese 177, pp. 165-192.

Gillett, C. (2013). 'Understanding the Sciences through the Fog of "Functionalism(s)", in P. Huneman (ed.) Functions: Selection and Mechanisms. New York: Springer, pp. 159-181.

Hardin, C. L. (1988). Color for Philosophers. Indianapolis, IN: Hackett.

Jackson, F. (1982). 'Epiphenomenal Qualia,' Philosophical Quarterly 32, pp. 127-136.

Lewis, D. (1972). 'Psychophysical and Theoretical Identifications,' Australasian Journal of Philosophy 50, pp. 249-258.

Melnyk, A. (2006). 'Realization and the Formulation of Physicalism,' Philosophical Studies 131, pp. $127-155$.

Nagel, T. (1974). 'What Is it Like to be a Bat?' Philosophical Review 83, pp. 435-450.

Polger, T. (2007). 'Realization and the Metaphysics of Mind,' Australasian Journal of Philosophy 85, pp. 233-259.

Shoemaker, S. (1975). 'Functionalism and Qualia,' Philosophical Studies 27, pp. 291-315.

Shoemaker, S. (1981). 'Absent Qualia Are Impossible - A Reply to Block,' Philosophical Review 90, pp. 581-599.

Shoemaker, S. (2001). 'Realization and Mental Causation,' in C. Gillett and B. Loewer (eds) Physicalism and its Discontents. Cambridge: Cambridge University Press, pp. 23-33.

Shoemaker, S. (2007). Physical Realization. New York: Oxford University Press.

Teller, P. (1998). 'Quantum Mechanics and Haecceities,' in E. Castellani (ed.) Interpreting Bodies: Classical and Quantum Objects in Modern Physics. Princeton, NJ: Princeton University Press, pp. 114-141. 
White, B. (2017). 'Metaphysical Necessity Dualism,' Synthese. https://doi.org/10.1007/ s11229-016-1308-5.

Wilson, J. (1999). 'How Superduper Does a Physicalist Supervenience Need to be?' Philosophical Quarterly 50, pp. 33-52.

Wilson, J. (2011). 'Non-Reductive Realization and the Powers-Based Subset Strategy,' The Monist 94, pp. 121-154.

Wilson, J. (2016). 'Metaphysical Emergence: Weak and Strong,' in T. Bigaj and C. Wüthrich (eds) Metaphysics in Contemporary Physics. Brill: Leiden, pp. 345-402. 